\title{
The dynamics of root meristem distribution in the soil
}

\author{
L. DUPUY ${ }^{1}$, M. VIGNES ${ }^{2}$, B. M. MCKENZIE ${ }^{1} \&$ P. J. WHITE ${ }^{1}$
}

${ }^{1}$ Scottish Crop Research Institute and ${ }^{2}$ Biomathematics \& Statistics Scotland, Invergowrie, Dundee, DD2 5DA, Scotland, UK

\begin{abstract}
Plants must develop efficient root architectures to secure access to nutrients and water in soil. This is achieved during plant development through a series of expansion and branching processes, mostly in the proximity of root apical meristems, where the plant senses the environment and explores immediate regions of the soil. We have developed a new approach to study the dynamics of root meristem distribution in soil, using the relationship between the increase in root length density and the root meristem density. Initiated at the seed, the location of root meristems in barley seedlings was shown to propagate, wave-like, through the soil, leaving behind a permanent network of roots for the plant to acquire water and nutrients. Data from observations on barley roots were used to construct mathematical models to describe the density of root meristems in space. These models suggested that the morphology of the waves of meristems was a function of specific root developmental processes. The waves of meristems observed in root systems of barley seedlings exploring the soil might represent a more general and fundamental aspect of plant rooting strategies for securing soil resources.
\end{abstract}

Key-words: architecture; development; dynamics; meristem; model; root soil interaction; wave.

\section{INTRODUCTION}

Land plants grow in soil where water and mineral nutrients are heterogeneously distributed. Plant survival, growth and fecundity are largely conditioned by the ability to acquire these resources effectively (Aerts, Boot \& van der Aart 1991). The architecture of a plant's root system affects its ability to access these resources, and there is considerable evidence linking root architectural properties with the efficient acquisition of water and nutrients (Hodge et al. 1999; White et al. 2005; Walk, Jaramillo \& Lynch 2006; Lynch 2007). However, the fundamental mechanisms controlling the development of root architectures and acclimation to the prevailing environmental conditions is complex and poorly understood.

Root architecture results from the activity of apical meristems and is produced by a sequence of expansion and lateral initiation events at the proximity of root apices. Newly created roots are placed rigidly in the soil and the final form of the root system is a direct consequence of the

Correspondence: L. Dupuy. Fax: +44(0)1382 562426; e-mail: lionel.dupuy@scri.ac.uk patterns of root expansion and lateral root initiation in the proximity of root tips. Because mature roots are immobile, it is essential that meristematic activity is controlled and coordinated in conjunction with local soil properties. For example, the efficient acquisition of water and essential mineral nutrients requires an ability to detect resource-rich patches and concentrate growth within these patches (Zhang \& Forde 1998; Wilkinson \& Davies 2002; White et al. 2005; Hodge 2009).

How a plant controls the behaviour of its meristems is therefore crucial for understanding the plasticity of root architecture. The detailed mechanisms by which water and the availability of mineral nutrients are sensed by plants remain poorly understood. However, there is increasing evidence that the sensing mechanisms are located at root apices (Amtmann et al. 2006). The sensing of external nitrate concentration has, for example, been found to be regulated by the $A N R 1$ gene, which is expressed in the root tip (Zhang \& Forde 1998, Remans et al. 2006, Forde \& Walch-Liu 2009). Similarly, a series of experiments by (Svistoonoff et al. 2007) has demonstrated that physical contact of the Arabidopsis root tip with phosphate is necessary and sufficient to arrest the growth of primary roots, and that Low Phosphate Root (LPR) genes are involved in this response.

Unfortunately much less is known about how root system architectures are generated through the distribution and activity of root meristems. This article reveals how root meristems are distributed and explore the soil, and how this can be constrained by specific developmental processes. Firstly, we developed a new approach to identify regions of meristematic activity in the soil based on the mathematical relationship between an increase in root length density and the root meristem density. Data from a minirhizotron experiment monitoring root system development of barley seedlings were then used to construct mathematical models describing the density of root meristems in space. We show that peaks of meristem abundance propagate in space like waves, and that developmental processes can influence the morphologies of these waves.

\section{MATERIAL AND METHODS}

\section{Experimental setup}

Barley (Hordeum vulgare L.) cv. Optic was grown in soil in concrete bins in a vented glasshouse. The bins were $1.75 \mathrm{~m} \times 1.25 \mathrm{~m} \times 0.66 \mathrm{~m}$, with drainage at the base. The depth of the soil in the concrete bins was comparable to the growing depth of roots in fields at the Scottish Crop 


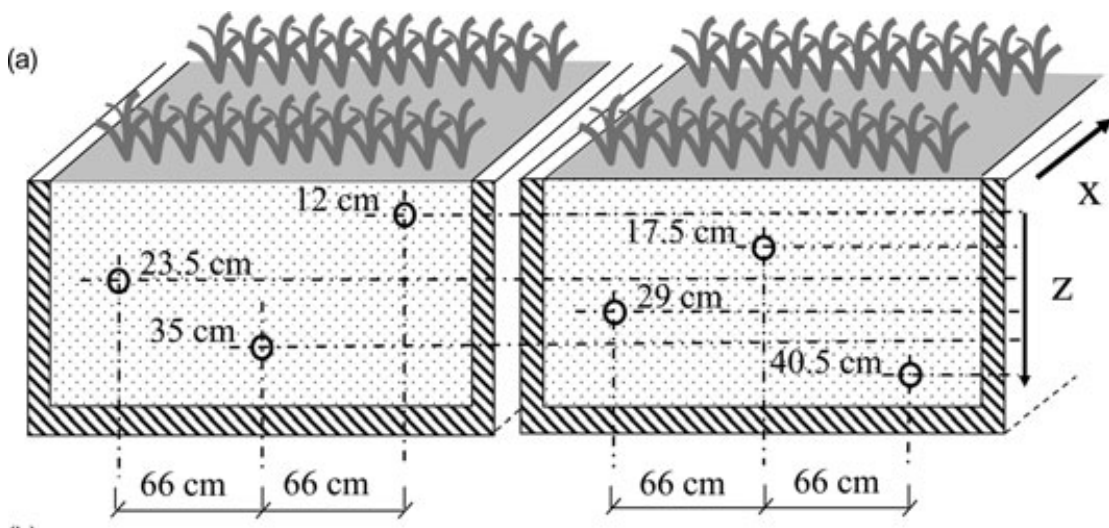

(b)

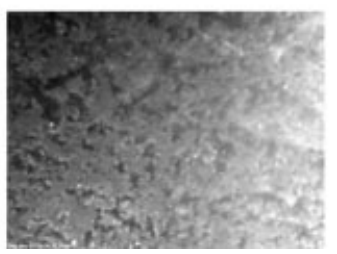

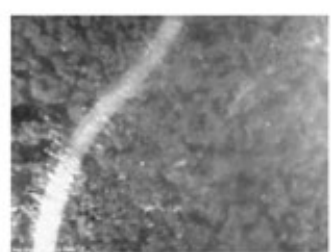

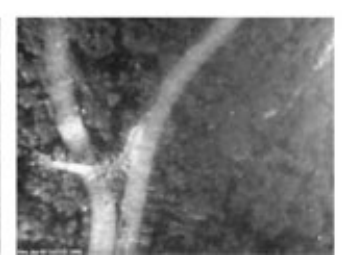

Figure 1. (a) Experimental system. Transparent minirhizotron tubes were placed at 6 different depths in two distinct concrete bins. Barley cv. Optic was grown in four rows at a distance of $32 \mathrm{~cm}$ from each other. Rows were sown perpendicular to the axes of the tubes to allow measurements of root intercepts from four different rows at each depth. (b) Camera images taken from a minirhizotron tube showing the root intercepts at the same position at $3 \mathrm{~d}$ intervals.
Research Institute. The bins were filled with soil in layers. Clear plastic minirhizotron access tubes were placed horizontally across the width of each concrete bin (on the soil surface as each layer was packed). The tubes were $1.25 \mathrm{~m}$ long and had a diameter of $50 \mathrm{~mm}$. Two bins were used for the experiment. We used three minirhizotron tubes in each bin and obtained measurements at six different depths (Fig. 1a) on a daily basis over $9 \mathrm{~d}$.

The soil was, from the top, $100 \mathrm{~mm}$ of Bullionfield at the Scottish Crop Research Institute, Scotland, UK $\left(56^{\circ} 27^{\prime} \mathrm{N}\right.$ $\left.3^{\circ} 40^{\prime} \mathrm{W}\right)$. The soil is Carpow Series, a Stagnic Cambisol derived from undifferentiated sandstone, with composition: sand $710 \mathrm{~g} \mathrm{~kg}^{-1}$, silt $190 \mathrm{~g} \mathrm{~kg}^{-1}$ and clay $100 \mathrm{~g} \mathrm{~kg}^{-1} ; \mathrm{pH}\left(\mathrm{H}_{2} \mathrm{O}\right)$ $6.2,1.9 \% \mathrm{C}$ and $0.07 \%$ N. Prior to sowing, the soil in the bins was repeatedly irrigated until drainage water occurred. This irrigation not only aided the packing of the soil but also ensured that the soil was at field water capacity for sowing. The bulk density of the soil was $1.18 \mathrm{~g} \mathrm{~cm}^{-3}(+/-0.0008)$. As some settling occurred with the irrigation, extra soil was added as needed to maintain its depth.

Barley was sown in four rows at approximately 180 seeds $\mathrm{m}^{-2}$. The seeding rate was half that typically used by farmers in the UK to avoid overlapping of roots from distinct rows. Seeds were sown by making a groove approximately $10 \mathrm{~mm}$ deep in the soil surface and sprinkling the seed evenly into this groove. The groove was then covered with the displaced soil. An open dish of water was placed on a bench adjoining the concrete bins. The evaporation from this dish was measured three times per week. Irrigation was applied to the plants in order to replace evaporation from this open water surface $\left(2 \mathrm{~mm} \mathrm{~m}^{-2} \mathrm{~d}^{-1}\right)$. Seedlings were grown with ambient light ( $17 \mathrm{~h} 30 \mathrm{~min}$ day length) at a minimum temperature of $7.5^{\circ} \mathrm{C}$ and maximum temperature of $19.5^{\circ} \mathrm{C}$.

A Quickcam Express ${ }^{\circledR}$ camera, Logitech (Fermont, CA, USA) was used to collect images from the minirhizotron.
The camera was inserted horizontally into each of the tubes every day and images were captured every $1 \mathrm{~cm}$ along the tube to capture root intercepts (i.e. roots in contact with the tube). Images were $1.2 \mathrm{~cm} \times 0.8 \mathrm{~cm}$ in size. This provides suitable resolution to identify living roots accurately (Fig. 1b). The experiment was started $8 \mathrm{~d}$ after sowing when a few root intercepts were observed from the shallowest tube, and was stopped $9 \mathrm{~d}$ later, when a few root intercepts were recorded from the deepest tubes. In this study, and throughout the modelling, we ignored root mortality, although the following theory could be extended to address this issue through appropriate terms in Eqns 1-3. This simplification is justified as we are considering young plants, and because no root decay was observed in any images.

The lengths of root segments intercepting each minirhizotron tube were measured semi-automatically from digital images. Software was developed to process the large number of images more rapidly. All root segments were traced manually on a digital image. The software then generated files containing coordinates defining the spatial location of each root (http://www.archiroot.org.uk/ doku.php/navigation/architools). The depth and distance from the planted row were defined by the depth of the tube and the position of the camera within the tube, respectively. Smoothed root length density distributions were calculated from the spatial location and lengths of root segments using a Hanning filter function from the python SciPy library (http://www.scipy.org/) with a window of $7 \mathrm{~cm}$.

\section{Definition of the root system}

Root architectures are complex networks of interconnected branches, described by their geometrical (e.g. root size, position and direction of growth) and topological (e.g. developmental relationships between roots) properties. The geometrical and topological properties of plant 


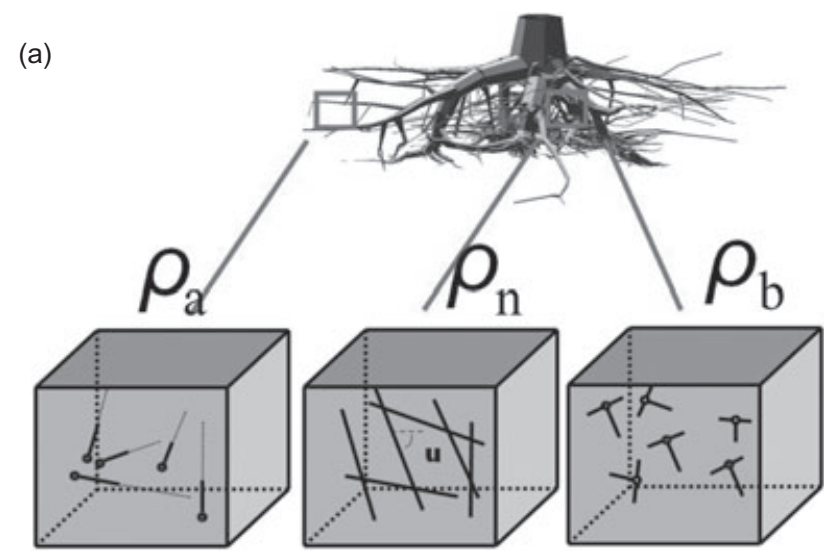

(b)

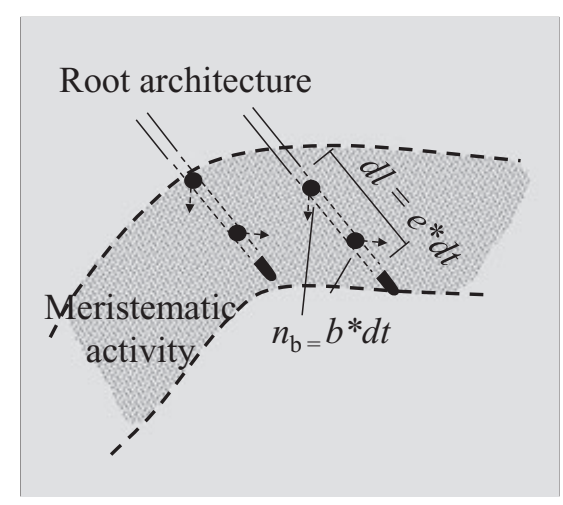

(c)

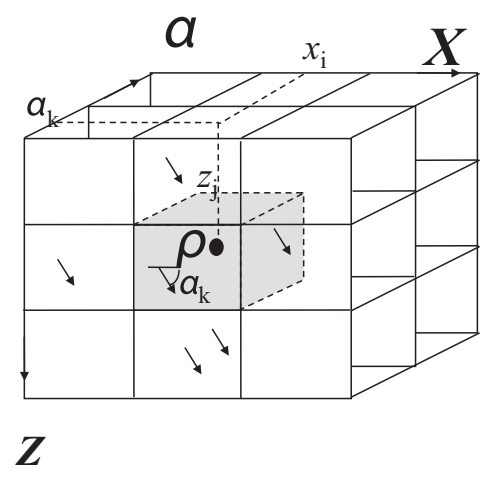

Figure 2. (a) Root system architecture is described by three density distributions: the root apical meristem density distribution (denoted $\rho_{\mathrm{a}}$ ), the root length density distribution (denoted $\rho_{\mathrm{n}}$ ), and the root branching density distribution (denoted $\rho_{\mathrm{b}}$ ). (b) The dynamics of the root system is determined by meristematic activity. During a time increment $d t$, each root will increase its length $l$ proportionally to the expansion rate $e$ : $d l=e^{*} d t$. In a unit volume, the total length of root created per unit time is therefore $e^{*} \rho_{\mathrm{a}}$. Similarly, the number of branching points along the root created per unit time in a unit volume is $b$. (c) A finite volume method was used to solve Eqn 4. This method used a grid to divide the root zone into control volumes or elements. Each element was associated with a node defined by its position in the grid $\left(x_{\mathrm{i}}, z_{\mathrm{j}}, \alpha_{\mathrm{k}}\right)$ and the value of the root meristem density at this point. Fluxes of roots entering and leaving each element were determined and these were used to calculate the evolution of the root meristem density distribution incrementally. architectures can be defined mathematically as discrete structures made of connected segments and vertices (Godin, Costes \& Sinoquet 1999). These representations currently form the basis for many plant architectural models (de Reffye \& Houllier 1997). Previous studies by the authors (Dupuy et al. 2005b) have also established that equivalent information can be represented in the form of spatial root distributions, where both topological and geometrical properties are represented as density functions.

We chose to represent root structures as density functions because these allow a formal description of the relationships between the dynamics of meristem distribution and root architectures (Bastian et al. 2008). Here, a plant's root system is defined by three density distributions: the root length density (denoted $\rho_{\mathrm{n}}$ ) defines the geometrical properties of root architecture, the plant topology is defined through the root branching density (denoted $\rho_{\mathrm{b}}$ ) and the root apical meristem density (denoted $\rho_{\mathrm{a}}$ ) indicates regions of primary growth. Because densities can be general multivariate functions, they can produce precise descriptions of root architecture. By including the direction of root growth in the definitions of density, it is possible to produce a more exact description of root morphology (Grabarnik, Pagès \& Bengough 1998; Dupuy et al. 2005b).
Densities may vary according to depth, incline angle of individual roots and time (respectively $z, \alpha, t)$. The root meristem density $\rho_{\mathrm{a}}(z, \alpha, t)$ is such that $\int_{z 1<z<z 2} \int_{0<\alpha<\pi} \rho_{a}(z, \alpha, t) \cdot d \alpha d z$ represents the number of root apical meristems at time $t$, between depths $z_{1}$ and $z_{2}$, and whose growth direction is contained between 0 and $\pi$. In the following paragraphs, we will consider only two physical dimensions, $x$ (horizontal distance to the row) and $z$ (depth), and the incline angle of roots $(\alpha)$ (Fig. 2). Therefore, the units in two-dimension (2D) of $\rho_{\mathrm{a}}$ and $\rho_{\mathrm{b}}$ are $\mathrm{cm}^{-2}$ and the unit of $\rho_{\mathrm{n}}$ is $\mathrm{cm}^{-1}$.

Root length and branching density distributions evolve through time as a function of root expansion rate $e\left(\mathrm{~cm} \mathrm{~d}^{-1}\right)$, branching rate $b\left(\mathrm{~cm}^{-2} \mathrm{~d}^{-1}\right)$ and tropic rate $g\left({ }^{\circ} \mathrm{d}^{-1}\right)$. Although each density distribution describes independent aspects of root architecture, they all result from the functioning of meristems, which can be expressed mathematically as:

$$
\begin{aligned}
& \frac{\partial \rho_{\mathrm{b}}}{\partial t}=b \quad \text { Volumetric root branching rate }\left(\mathrm{cm}^{-2} \mathrm{~d}^{-1}\right) \\
& \frac{\partial \rho_{\mathrm{n}}}{\partial t}=e \rho_{\mathrm{a}} \quad \text { Volumetric root production rate }\left(\mathrm{cm}^{-1} \mathrm{~d}^{-1}\right)
\end{aligned}
$$


These mathematical equations imply that the increase in branching density and root length density is a function of root meristem density (Fig. 2a). The quantities $e \rho_{\mathrm{a}}$ and $b$, denote the length of root and number of branches being produced per day per unit volume, respectively. These quantities are determined by rates of apical meristem production, cell production and cell expansion at root apices, which we will term 'meristematic activity'. Meristem activity is linked to the time derivative of the root length density (Eqn 2) and can be determined directly from time sequences of the root length density distributions. Equations 1 and 2 also imply that both total root length density and branching density distributions are determined by the timecourse of meristem activity. Therefore, the architecture of root systems can be viewed as the footprint of meristem activity (Fig. 2a).

\section{Modelling the dynamics of root growth}

To explain the experimentally observed dynamic patterns of root meristem activity, we have built a mechanistic model that describes how the density distribution of root apices evolves as a function of the root expansion rate, gravitropism and branching rate (see Supporting Information). The general equation underlying this model is:

$$
\frac{\partial \rho_{\mathrm{a}}}{\partial t}+\nabla^{*} \cdot\left(\rho_{\mathrm{a}} g\right)+\nabla \cdot\left(\rho_{\mathrm{a}} e u\right)=b
$$

The change in the number of apices (or meristems) at a given location $r=(x, z)$ and with a given growth direction $u=(\cos \alpha, \sin \alpha)$ results from the number of roots entering the location from upstream regions $(r-e u d t, u)$, the number of roots leaving the location at expansion rate $e\left(\mathrm{~cm} \mathrm{~d}^{-1}\right)$, the number of roots changing their orientation through gravitropism $g\left(\mathrm{~d}^{-1}\right)$ and the creation of new meristems through branching rate $b\left(\mathrm{~d}^{-1}\right)$. It is logical then that the gradient operators appearing in the general equation are $\nabla$ (gradient operators for the spatial coordinates) and $\nabla *$ (gradient operators for the angle coordinates).

This model must be adapted for roots of different branching order by combining the meristem densities of each branching order $i, \rho_{\text {ia. }}$. Each density function obeys the same conservation law (Eqn 3) but has different growth parameters $(g, e, b)$. In particular, the initiation of new meristems of branching order $i$ is proportional to the meristem density of branching order $i-1$, as the development of a root system with two different branching orders can be written as:

$$
\frac{\partial \rho_{\mathrm{ia}}}{\partial t}+\frac{\partial \rho_{\mathrm{ia}} g_{2}}{\partial \alpha}+\frac{\partial \rho_{\mathrm{ia}} e_{\mathrm{i}} \cos \alpha}{\partial x}+\frac{\partial \rho_{\mathrm{ia}} e_{\mathrm{i}} \sin \alpha}{\partial z}=b_{\mathrm{i}}, i \in\{1,2\}
$$

The two parts of Eqn 4 are coupled through the source term $b_{2}$, which is a function of $\rho_{1 \mathrm{a}}$. The terms $e, g$ and $b$ in Eqns 3 and 4 are not constants in general but functions that encode both developmental behaviour and root/soil interactions. We used the following growth functions to model meristem density:

$$
\begin{aligned}
& e_{1}=e_{2} s=c t e \\
& g_{1}=g_{2} s^{2}=g_{11}(\pi / 2-\alpha) \\
& b_{1}=0 \\
& b_{2}=b_{21}\left(\rho_{1 \mathrm{a}}\left(r, u+b_{22}, t\right)+\rho_{1 \mathrm{a}}\left(r, u-b_{22}, t\right)\right) / 2+b_{23} \rho_{2 \mathrm{n}}
\end{aligned}
$$

$s$ is the scaling factor between the growth of the roots of branching order 1 and $2, e_{1}$ and $e_{2}$ are constant (in space and time) root expansion rates, $g_{1}$ and $g_{2}$ are the rates at which root apices orientate verticality, $b_{21}$ is the branching rate, $b_{22}$ is the branching angle and $b_{23}$ is the adventitious branching rate (lateral root initiation on mature tissues).

\section{Analysis of the dynamics of meristem activity}

The apical meristem of a root is confined to a small region at the root apex. Although apical meristems can be observed in images taken from minirhizotrons, they are present at the surface of the minirhizotron for a short period only. Therefore, the estimation of their density distribution from direct observations is less accurate than the estimation of their density distribution from the footprint of their activity, the root length density.

Density distributions of root apical meristems can be estimated from root length density distributions using Eqn 2, which states that temporal changes in root length density are mechanistically related to root meristem activity. The analysis was carried out using data from each minirhizotron tube to characterize meristem activity at a given depth. Two indicators of the bulk spatial distribution of meristem activity in soil $I_{\mathrm{a}}$ and $I_{\mathrm{g}}$ were used to characterize the development of the root system,

$$
I_{\mathrm{a}}(z, t)=\iint_{x \alpha} \dot{\rho}_{\mathrm{n}}(x, z, \alpha, t) d x
$$

the intensity of root meristem activity $\left(\mathrm{cm} \mathrm{d}^{-1}\right)$ and

$$
I_{\mathrm{g}}(z, t)=I_{\mathrm{a}} \times \frac{\left(x_{\mathrm{g}}-x_{\mathrm{c}}\right)}{x_{\mathrm{c}}}
$$

the position of meristem activity $\left(\mathrm{cm}^{2} \mathrm{~d}^{-1}\right)$.

$I_{\mathrm{a}}$ is therefore the area between two consecutive root length density curves and is a measure of the intensity of root meristem activity at a given time. $I_{\mathrm{g}}$ is a measure of the offset of meristems along the $x$ axis. $x_{\mathrm{g}}(t)$ is the centre of mass of $\dot{\rho}_{\mathrm{n}}$ and $x_{\mathrm{c}}(t)$ is the geometric centre. Because the regions of meristem activity evolve with time, we chose to measure the offset as the position of the centre of mass relative to the geometric centre $x_{\mathrm{c}}$. This position is then multiplied by $I_{\mathrm{a}}$ to normalize the offset. By analogy to a mechanical system, $I_{\mathrm{a}}$ represents the total weight of meristems distributed along the horizontal axis, while $I_{\mathrm{g}}$ represents the bending moment due to the position of the application of this weight. Plots representing the 
trajectories of experimental data and model predictions in the $\left(I_{\mathrm{a}}, I_{\mathrm{g}}\right)$ space were used to analyse meristem activity.

\section{Analytical model of the dynamics of root meristem density}

In order to make predictions of the dynamics of root meristem density, it is useful to have a simple analytical model. This was achieved by solving Eqn 4 using the method of characteristics (Meister \& Struckmeier 2002). This is a technique for solving a partial differential equation by decomposing it into two ordinary differential equations that can be solved. A one-dimensional (1D) solution to Eqn 4 was obtained (Supporting Information) and can be written as:

$\rho_{\mathrm{a}}(z, \alpha, t)=c(1+b t) N_{0, \mathrm{v}}\left(z-e t-e \alpha^{2} / 4 g\left(e^{-2 \mathrm{gt}}-1\right), \alpha\right)$

where $N_{0, \mathrm{v}}$ is the normal distribution of mean 0 and variance $\mathrm{v}$. This $1 \mathrm{D}$ analytical model predicts that maxima of meristem density are formed and that such maxima travel under steady-state conditions, at the same speed as the individual root expansion rate $e$. In the following section, we will refer to this phenomenon as a 'meristematic wave', which defines the propagation of the maxima of root meristem density in space and time (French 1971).

The analytical model was fitted to the experimental data using a downhill simplex algorithm (Avriel 2003), a nonlinear optimization algorithm implemented in the python SciPy library (http://www.scipy.org/). The result of the optimization provided individual root developmental parameters $(g$, $e, b)$ to compare the model predictions against the root meristem density distributions observed experimentally.

\section{Numerical simulation of root density distributions}

The finite volume method (Leveque 2002; Heinen, Mollier \& De Willigen 2003) was used to obtain approximate (numerical) solutions to Eqn 4. This method uses a grid to divide the root zone into control volumes, termed elements. Each element is associated with a node defined by its position in the grid. Nodes are a finite set of coordinates $\left(x_{\mathrm{i}}, z_{\mathrm{j}}\right.$, $\alpha_{\mathrm{k}}$ ) at the centre of the elements of the grid (Fig. 2b). The numerical simulation then uses the root meristem density at each node to compute the fluxes of root meristems entering and leaving each element using Eqn 4. This enables the evolution of root density distributions in soil to be developed incrementally from a given set of initial conditions. To compute the fluxes of root meristems between elements, each coordinate was treated separately and an upwind scheme method was implemented (Leveque 2002). This method used the instantaneous root meristem density of adjacent elements and the local direction of root growth to calculate the fluxes into an element. For simulations, the total soil volume was assumed to be $30 \mathrm{~cm}$ by $40 \mathrm{~cm}$ by $\pi$. This was divided into $30 \times 40 \times 40=48000$ elements to accommodate the spatial coordinates $(x, z)$ and the root angle $\alpha$ in Eqn 4. All simulations were initiated in the top
Table 1. Parameters used in numerical simulations of root length density and root meristem density distributions

\begin{tabular}{lll}
\hline & Mean value & Domain of variation \\
\hline$e_{1}\left(\mathrm{~cm} \mathrm{~d}^{-1}\right)$ & 2 & - \\
$g_{1}\left(\mathrm{~d}^{-1}\right)$ & 0.2 & $0.1-0.4$ \\
$b_{21}\left(\mathrm{~d}^{-1}\right)$ & 5 & $2.5-7.5$ \\
$b_{22}\left(\mathrm{~d}^{-1}\right)$ & $\pi / 6$ & $\pi / 12-\pi / 3$ \\
$b_{23}\left(\mathrm{~d}^{-1}\right)$ & 0.03 & $0.02-0.04$ \\
$s($ dimensionless $)$ & 1.5 & $1-2$ \\
Duration (d) & 8 & - \\
Time increment $(\mathrm{d})$ & 0.04 & - \\
\hline
\end{tabular}

left corner of the soil volume $(x, z=0,0)$. The density of root meristems in boundary elements outside the simulated soil volume were defined as the mirror image of the boundary elements inside the soil volume to preserve a vertical plane of symmetry.

We have confirmed that the numerical simulations described here produce the same root meristem distributions and root length distributions as the simple architectural model of Dupuy, Fourcaud \& Stokes (2005a), which simulates root development using equivalent parameters $(g, e, b)$ to Eqn 4. A sensitivity analysis of the numerical model described by Eqn 4 was performed by varying the developmental parameters for barley roots (Table 1 ). Fifteen simulations were run, with each of the parameters taking successively average, low and high values (while other parameters were kept at their mean values). These simulations provided insight to how root length density distributions and root meristem density distributions might be altered by factors affecting root gravitropism $(g)$, rate of expansion $(e)$ and rate of lateral branching $(b)$.

The simulation program was written using the python programming language and the code can be freely downloaded at http://www.archiroot.org.uk/doku.php/ navigation/architools.

\section{RESULTS}

\section{Minirhizotron data indicates that root meristem distribution has both spatial and temporal patterns}

Root intercepts were recorded at each minirhizotron tube for each of the $9 \mathrm{~d}$ of measurements, and root length density distributions were determined. Root intercepts were first observed at the tube closest to the soil surface and then at successively deeper tubes (Fig. 3). Variations in root length density were observed along all tubes (Fig. 3). Changes in the root length density distribution with time followed apparently similar patterns at each tube (Fig. 3): (1) the root length density increased with time; (2) the first root intercepts appeared close to the row of barley plants and, subsequently, root intercepts were observed at greater distances from the row; (3) maxima of root length density were always observed where the first roots initially 


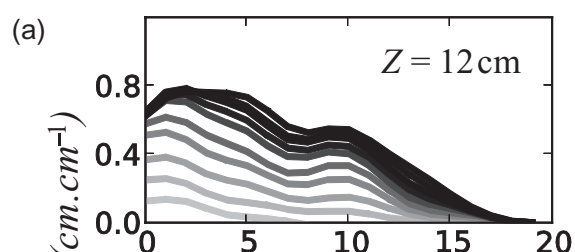

(c)

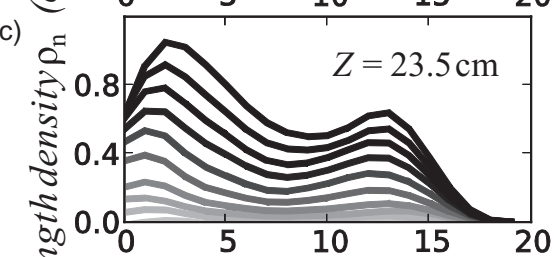

(e)

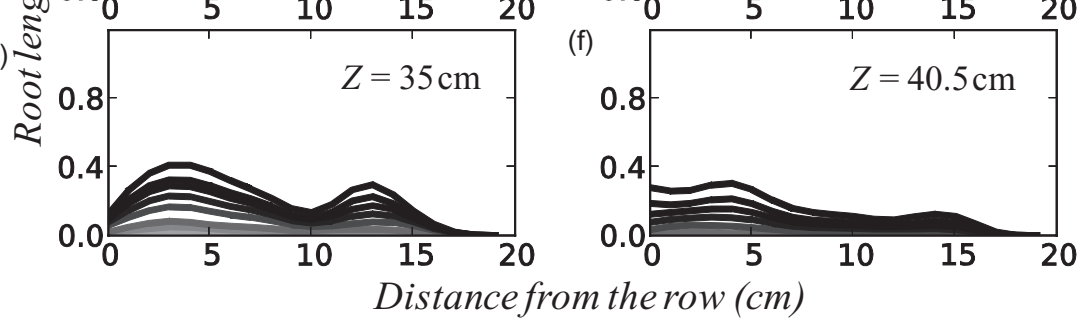

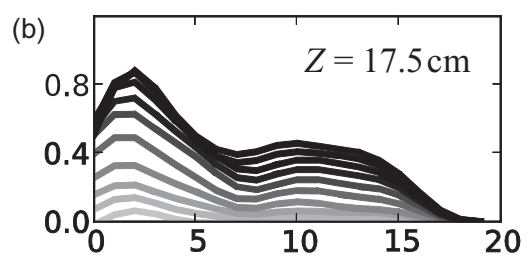

(d)

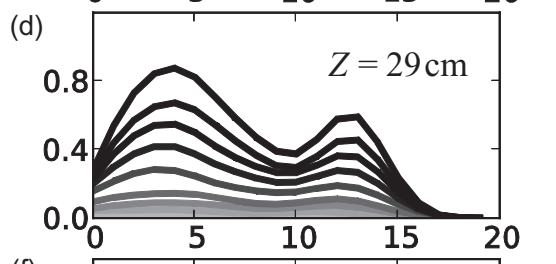

(f)

足

3. Temporal patterns of root length density distribution. Daily measurements of root length density were started $9 \mathrm{~d}$ after sowing (referred to as day $0)$. Root length density distribution $\left(\mathrm{cm} \mathrm{cm}^{-1}\right)$ constructed for each minirhizotron tube and for each day of the measurement (day 0 to day 8). (a-e) The reconstructed root length densities at, respectively, 12, 17.5, 23.5, 29, 35 and $40.5 \mathrm{~cm}$ depths. Colours correspond to measurement day. appeared; and (4) local maxima of root length density developed at distances further away from the row with time. A similar decrease in root length density with distance from the row has been reported for maize grown in the field (Grabarnik et al. 1998).

The changes in meristem activity during the experiment were summarized by plotting the two indicators, intensity of meristem activity $\left(I_{\mathrm{a}}\right)$ and position of meristem activity $\left(I_{\mathrm{g}}\right)$. During seedling growth, the curves of root meristem activity take the form of trajectories in the $I_{\mathrm{a}}, I_{\mathrm{g}}$ space (Fig. 4). The path followed by root meristem activity illustrates the changes in root length density distributions observed at each tube (Fig. 3). When there are no root intercepts at a tube, the $I_{\mathrm{a}}, I_{\mathrm{g}}$ coordinates are 0,0 . Because root meristem activity is distributed asymmetrically, and more root intercepts are first observed closer to the row, the position of meristem activity $\left(I_{\mathrm{g}}\right)$ is initially shifted to the left at all tubes (Fig. 4). As the root system develops, the intensity of root meristem activity $\left(I_{\mathrm{a}}\right)$ increases to a maximum. After this maximum is reached, at a position roughly below the row, the position of meristem activity is shifted to the right, as more intercepts are observed further away from the row. Eventually, meristem activity at a tube disappears, and the trajectory of root meristem activity returns to the origin $(0,0)$. Similar trajectories of root meristem activity are observed in all tubes. However, because root intercepts are first observed in shallower tubes, and subsequently in deeper tubes, the trajectories of root meristem activity at

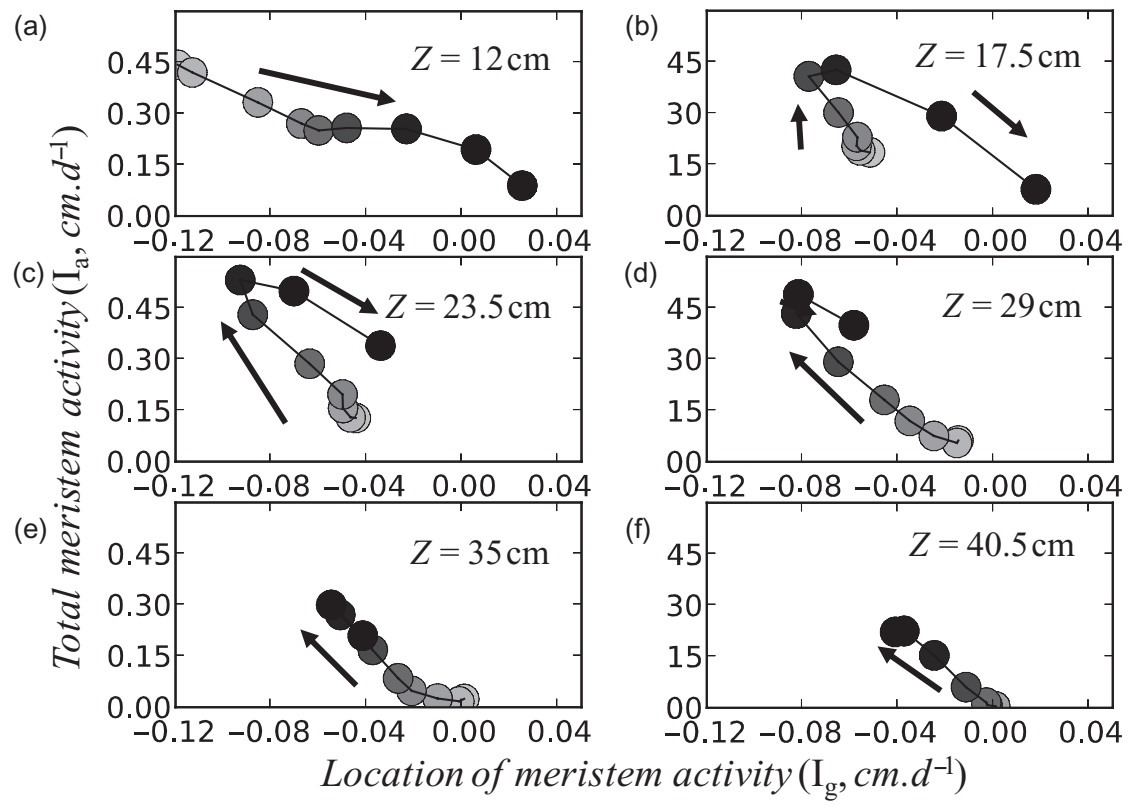

Figure 4. Meristem activity in soil during barley growth: two types of indicators (cf. Eqns 1 \& 2) were used to analyse the patterns of meristem activity during growth. $I_{\mathrm{g}}$ represents the position of the bulk meristems activity along the row ( $x$ axis), and $I_{\mathrm{a}}$ represents the total meristem activity ( $y$-axis). Plots show $\left(I_{\mathrm{g}}, I_{\mathrm{a}}\right)$ trajectories at $12,17.5,23.5,29,35$ and $40.5 \mathrm{~cm}$ depth (a-f, respectively). The same movement appears to propagate through depth during growth: the bulk of the meristem activity appears directly beneath the plant rows. After a maximum meristem activity is reached, the position of meristem activity apparently shifts from the row before disappearing. 
(a)

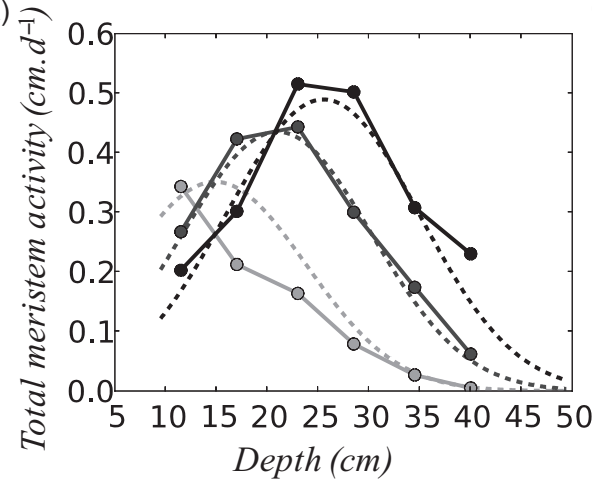

(b)

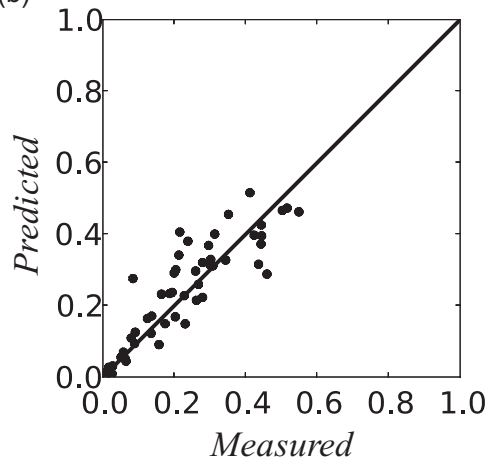

Figure 5. The one-dimensional analytical model shows that meristems form a peak of activity and that this peak propagates downward. (a) Comparison between model predictions and measurements of root meristem activity as a function of depth at $t=0,4$ and $8 \mathrm{~d}$ after measurements began, respectively, green brown and dark red colour, (solid) experimental and (broken line) model predictions (with $e=2.5$, $g=0.5, b=0.1$ ). (b) Predicted versus measured root meristem activity $\left(R^{2}=0.85\right)$. The solid line is the line $y=x$. each tube are asynchronous. In our experiments, only the trajectory after maximum root meristem activity was observed at the shallowest tube, while only trajectories prior to maximum root meristem activity were observed at the two deepest tubes. These observations indicate that the time required to reach maximum meristematic activity increases with depth, which suggests that maximum meristematic activity could propagate through the soil as a travelling wave.

\section{Models show that maximum root meristem activity propagates like a wave}

We have derived a general equation relating root growth processes to the distribution of root meristem density in the soil (Eqn 3). This equation is categorized as a hyperbolic partial differential equation. Interestingly, such equations occur frequently in physics, where they are linked to diverse forms of wave phenomena in acoustics, mechanics or electromagnetism (Leveque 2002; Meister \& Struckmeier 2002).

The analytical model (Eqn 8) also predicted that maxima of meristem activity were formed, and that these maxima travelled, at steady state, at the speed of the average root expansion rate $e$ (see Materials and Methods section) The analytical model was fitted to the experimental data and showed good agreement (Fig. 5). The fitting also provided information on developmental parameters for roots of the barley seedlings studied in our experiments. The root expansion rate, obtained using Eqn 8, was $e=2.2 \mathrm{~cm} \mathrm{~d}^{-1}$. This value is similar to those reported in earlier studies $\left(\approx 1.5-2 \mathrm{~cm} \mathrm{~d}^{-1}\right)$ for seminal roots of older plants (Drew 1975; Robinson 1996). The root branching rate, obtained using Eqn 8 , was $b_{2}=0.2 \mathrm{~d}^{-1}$. This value is one order of magnitude lower than the branching rates reported previously in the literature (Hackett 1971; Rose 1983). This is probably because the analytical model did not take into account that secondary and tertiary roots have slower expansion rates than primary roots (Rose 1983), which influences the fitted root branching rate indirectly. The gravitropic rate, obtained using Eqn 8, was $0.1 \mathrm{~d}^{-1}$. At an angle of $40^{\circ}$, this is equivalent to a rotation of $5^{\circ}$ per day, which is consistent with the values obtained on peas (Bonser, Lynch
\& Snapp 1996). The constant $c$ in Eqn 8 equalled $0.003 \mathrm{~cm}^{-1}$ and the variance $v$ equalled $14 \mathrm{~cm}$.

Simulations using the 2D numerical model expressed in Eqn 4 were compared with an explicit model of root architecture (Dupuy et al. 2005a). It was observed that their predictions of both root length density distribution and root meristem distribution were visually in good agreement (Fig. 6). Furthermore, the trajectories of meristem activity in the $\left(I_{\mathrm{a}}, I_{\mathrm{g}}\right)$ space showed similar features (see Fig. $6 \mathrm{c}-\mathrm{f}$ ), which supports the hypothesis that a wave of maximum meristematic activity (a meristematic wave) propagates through the soil.

\section{Root developmental processes can influence wave morphologies}

Numerical models were used to investigate the influence of root developmental processes on the dynamics of meristem activity. All numerical simulations produced meristematic waves, but distributions of root meristem density differed greatly. Poorly gravitropic roots (Fig. 7a) explored a greater volume of soil, but less intensively, than highly gravitropic roots (Fig. 7b). Increasing branching rate increased root length density proportionally (data not shown). A greater branching angle increased the volume of high meristem activity (data not shown). Exaggerating the difference in root expansion and gravitropism between seminal and secondary lateral roots $(s)$ caused regions of meristem activity to have a larger volume and to become more diffuse (Fig. 7c). Finally, adventitious branching produced permanent meristem activity within the root system (Fig. 7d).

\section{DISCUSSION}

\section{The meristematic wave - a new concept for understanding root structure/function relationships}

We have coined the term 'meristematic wave' to describe the propagation of maxima in root meristem activity through the soil (Fig. 8). The meristematic wave may be a fundamental architectural trait of plant root systems. The growth of an individual root occurs through cell division 


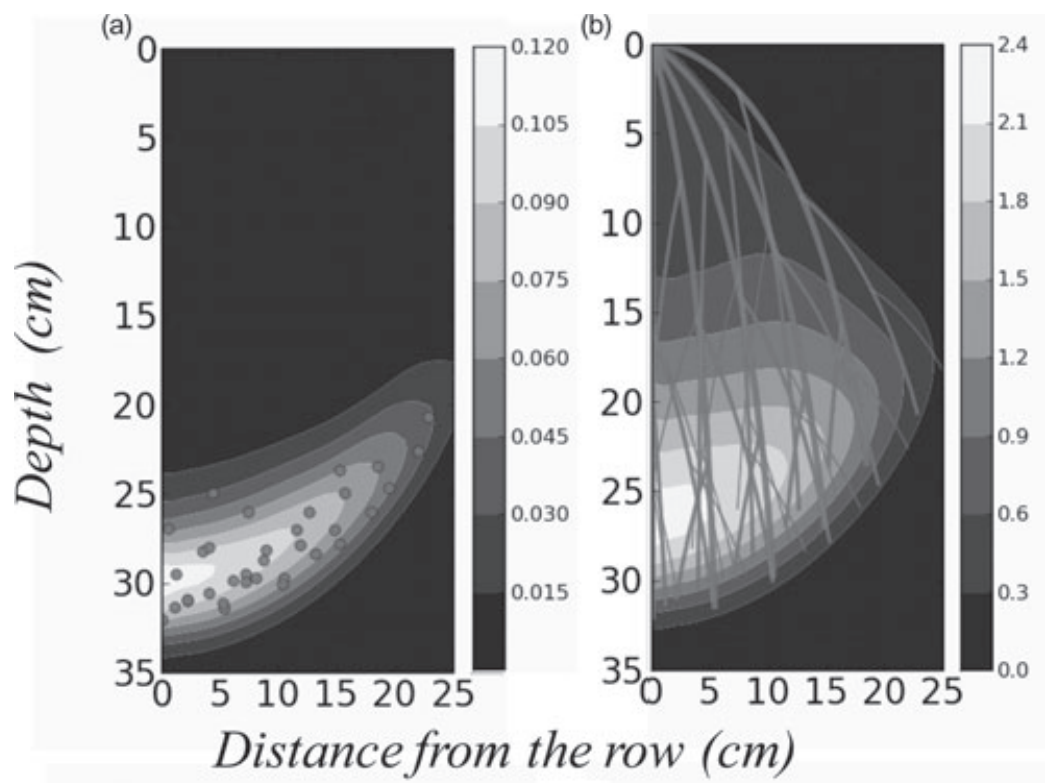

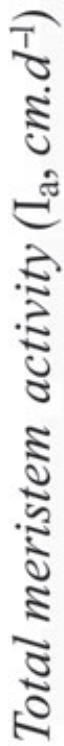

(d)

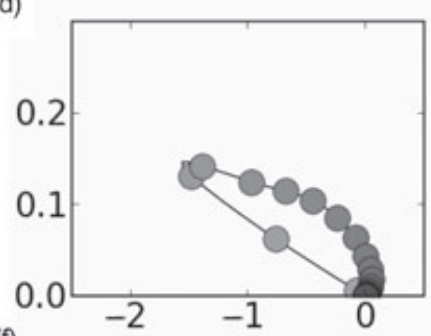

e)

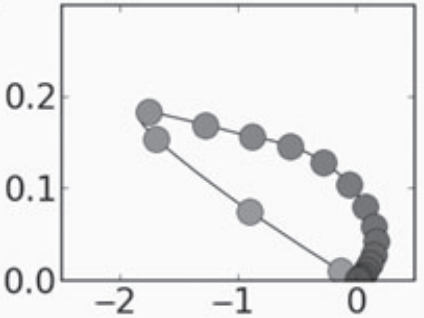

(f)

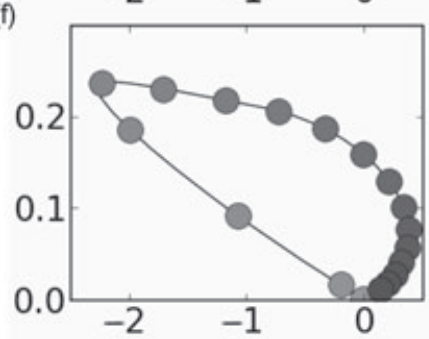

Figure 6. Numerical analysis of the model involved simulations for the period of $0-15 \mathrm{~d}$ : The results from the simulation of root meristem density and root length density as described in Eqns 1 and 2 (contour plot in a and b, respectively) were compared with meristem positions (circles in a) and whole root system (lines in b) from the architectural model using the same parameters. The trajectories of the position of the meristems in soil are visualized using the indicators $I_{\mathrm{a}}$ and $I_{\mathrm{g}}$ (Eqns 6 \& 7). (c-f) Trajectory plots at 10 , 15,20 and $25 \mathrm{~cm}$ depth, respectively. within the apical meristem and cell expansion close to the apical meristem. Newly created tissue eventually becomes rigid as cells differentiate (Beemster \& Baskin 1998; Chavarria-Krauser \& Schurr 2004). These developmental processes constrain the direction of advancing root growth and the permanent location of the root produced (Silk 2002). Single meristems are pushed by this indeterminate self-organized process, and the advancing meristems of several roots combine to constitute the simplest form of a meristematic wave. Our results demonstrate that the barley root system produces meristematic waves travelling through the soil (Figs 3-5). Our models and simulations suggest that meristematic waves can be produced by the repetition of simple developmental rules in individual roots (Fig. 7).

Plant roots must acquire resources distributed heterogeneously in the soil volume (Hodge 2004). Plant species employ diverse root system architectures to explore space and optimize resource acquisition (Fitter et al. 1991). Plants with highly tropic roots or with a smaller branching angle, explore space locally and can be more efficient in exploiting localized patches of resources in the soil (Ge, Rubio \& Lynch 2000). By contrast, the root systems of plants whose roots vary in their expansion rates, for example, through changes in diameter or in branching order (Pages 1995), or produce adventitious roots (Walk et al. 2006), proliferate more diffusely through the soil. Such spatial and temporal limitations to the generation of root system architectures are manifested in the contrasting strategies of plant species to intercept and exploit soil resources (Crick \& Grime 1987).

The region of highest root meristem activity defines the envelope of the soil volume being exploited intensively by a root system. This volume is of fundamental importance 
(a)

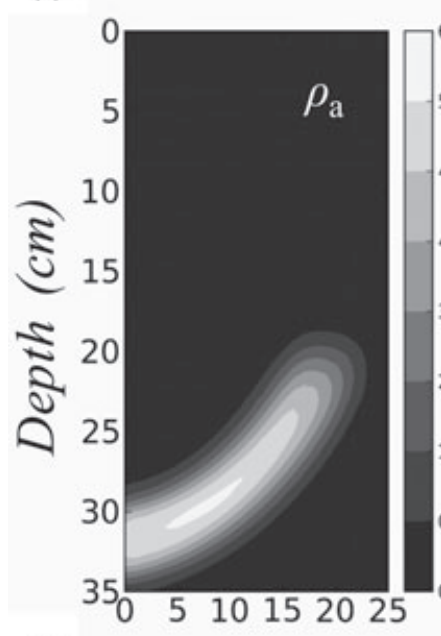

(c)

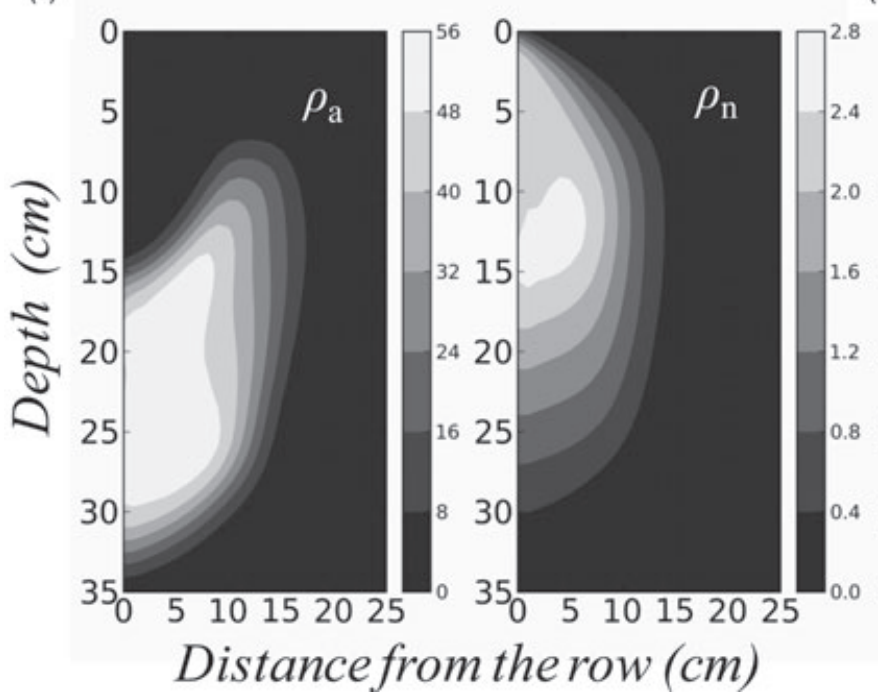

(b)

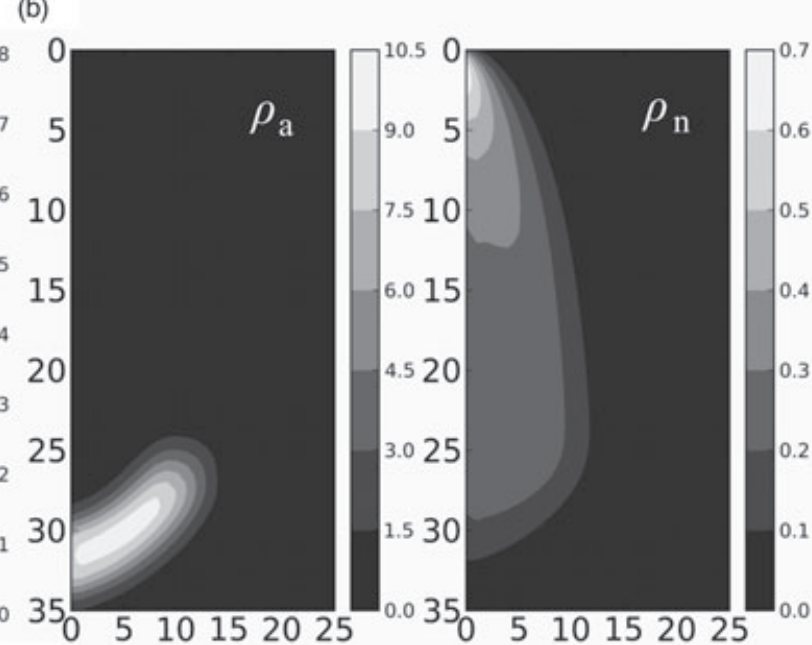

(d)

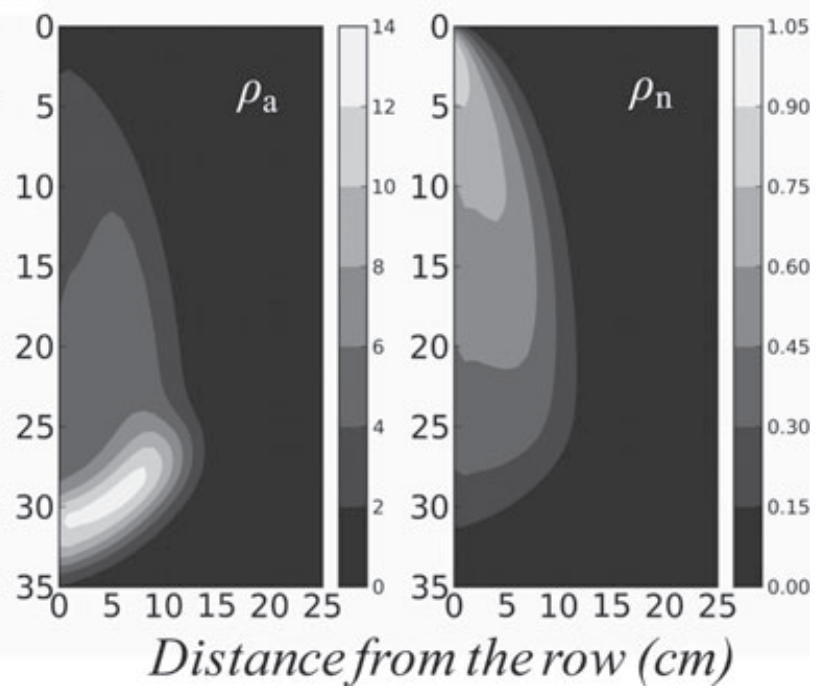

Figure 7. Influence of gravitropism (a, b) expansion rate of laterals $(c)$ and adventitious branching (d) on the patterns of root distribution in soil. Contours are meristem density functions and root length density functions with parameters $g_{11}=0.1 \mathrm{~d}^{-1}$ (a) and $g_{11}=0.4 \mathrm{~d}^{-1}(\mathrm{~b}), s=2(\mathrm{c})$ and $b_{23}=0.02 \mathrm{~d}^{-1}(\mathrm{~d})$. The remaining model parameters were kept constant: $b_{21}=0.2 \mathrm{~d}^{-1}, b_{22}=\pi / 6, b_{23}=0 \mathrm{~d}^{-1}$, $e_{1}=2 \mathrm{~cm} \mathrm{~d}^{-1}$ and $s=1$.

to resource acquisition. The ability of a plant to sense the availability of water, $\mathrm{N}$ and $\mathrm{P}$ resides close to the root apical meristem, which allows the development of lateral roots into resource-rich patches (Zhang \& Forde 1998; Wilkinson \& Davies 2002; White et al. 2005; Hodge 2009). The availability of mineral elements is often highest at the root apex (Claassen \& Barber 1974), and living root hairs, which contribute greatly to mineral uptake (Gilroy \& Jones 2000), are present predominantly in this region. The root apex is also the site of higher exudation of organic compounds (Badri \& Vivanco 2009), enzymes and mucilage (Morgan, Bending \& White 2005), increased microbial activity (Yang \& Crowley 2000; Nichol \& Silk 2001), and the apoplastic uptake of calcium and zinc (White 2001; White et al. 2002).

\section{A new approach to model the architecture of root systems}

Most architectural models predict root system development by simulating the incremental growth of independent tissues over time. In such models, the architecture of roots is explicit, and this allows complex analyses to be undertaken. For example, graph theory concepts can be used to analyse uptake efficiency (Fitter \& Stickland 1991), and physical models can be developed to study local interactions between roots and the soil (Clausnitzer \& Hopmans 1994; Doussan et al. 2006).

While explicit descriptions of root architectures are convenient to dissect biophysical, physiological and developmental processes, they have certain limitations. 


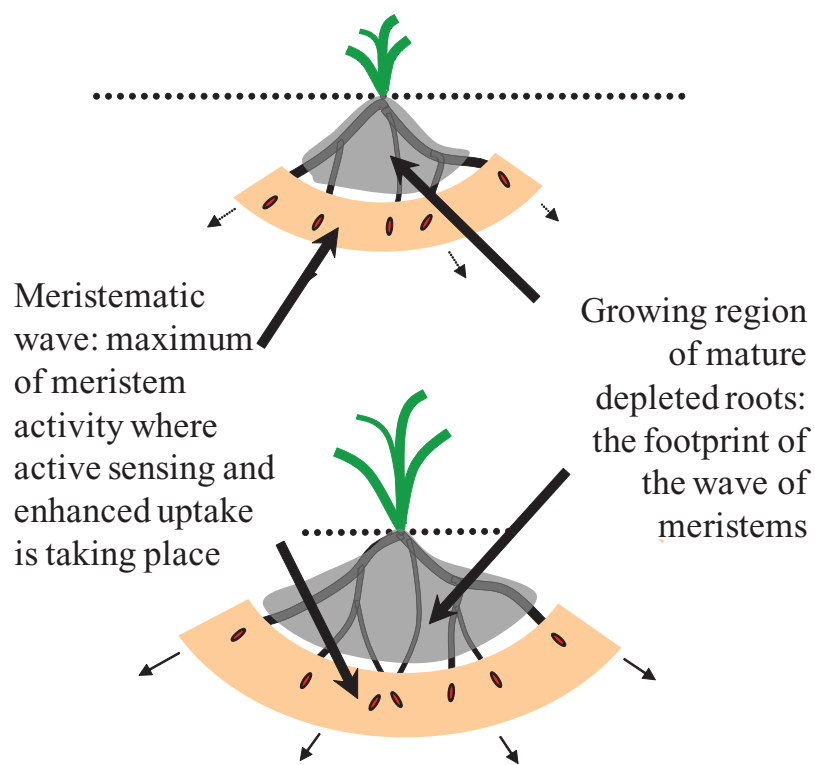

Figure 8. Model for whole plant root architectural trajectories.

Architectural models require accurate measurements of single organ properties, which make them difficult objects to parameterize (Jourdan, Rey \& Guédon 1995). The computational time required to generate the architecture of a plant root system depends on its size, and therefore, applications have mainly been restrained to the single plant level. Architectural models also generate complex structures and understanding their emergent properties can be difficult. Finally, architectural models produce a single and unique description of a root system for each simulation, which makes them unwieldy for studying the general properties of plant populations.

The root distribution models of Hackett and Rose (Hackett \& Rose 1972; Rose 1983), and subsequently, the reaction diffusion models (Gerwitz \& Page 1974; Acock \& Pachepsky 1996, Heinen et al. 2003), attempted to address these issues. However, none of these models explicitly incorporated root developmental processes. Describing root architecture in terms of diffusion incorrectly assumes that root expansion rate is proportional to gradients in root length density, and neither the root distribution nor the reaction diffusion models can describe how root length density, branching density, expansion rate and branching rate generate root system architectures.

We have formulated a mathematical framework to describe the development of root system architectures, in which the physiological properties, root apical meristem density, root length density and branching density are linked explicitly to developmental behaviours such as root expansion, initiation of new primordia and tropic responses. This framework can be used not only to construct new plant growth models but to extract key developmental information using classical experimental techniques such as minirhizotrons. The distribution of root meristem activity can be obtained from timecourse data of root length density using Eqn 2. The use of an analytical model (Eqn 8) allowed us to derive three important root developmental parameters (root expansion rate, root branching rate and root tropic rate) from images of root intercepts captured from minirhizotrons without the need for complete excavation of root systems. However, the models presented here are still at an early stage of their development. In the future, these models must be expanded to include phenomena such as differences in growth rates among root types, root mortality and local responses to environmental cues. Further research, combining improved image analysis and parameter estimation techniques, will allow the dynamics of root meristem activity and the development of root system architectures to be studied non-destructively.

\section{CONCLUSION}

The activity of root apical meristems is key to the development of root architectures in complex natural soil environments. Our work has demonstrated that live imaging using minirhizotrons can be used to obtain information on root meristem activity in soil. This can, in turn, be used to determine specific developmental properties of roots, including their expansion rate, branching rate and tropic rate. Both experimental observations and mathematical modelling have demonstrated that the development of a root system can be viewed as a propagating wave of meristematic activity and that root system architecture is the footprint of this process (Fig. 8). The next challenge is to extend these concepts to understand the consequences of phenotypic differences in the interactions between roots and their environment better.

\section{NOMENCLATURE}

$\begin{array}{ll}r(\mathrm{~cm}) & \text { Spatial coordinate } \\ z(\mathrm{~cm}) & \text { Depth } \\ x(\mathrm{~cm}) & \text { Distance from the row } \\ \alpha\left({ }^{\circ}\right) & \text { Incline angle defining direction of growth } \\ I_{\mathrm{a}}\left(\mathrm{cm} \mathrm{d}^{-1}\right) & \text { Change in root length } \\ I_{\mathrm{g}}\left(\mathrm{cm}^{2} \mathrm{~d}^{-1}\right) & \text { Change in position of root meristems } \\ \rho_{\mathrm{a}}\left(\mathrm{cm}^{-2}\right) & \text { Distribution of apical meristem density } \\ e \rho_{\mathrm{a}}\left(\mathrm{cm}^{-1} \mathrm{~d}^{-1}\right) & \text { Volumetric root production rate or } \\ & \text { 'meristematic activity' } \\ \rho_{\mathrm{n}}\left(\mathrm{cm}^{-1}\right) & \text { Distribution of root length density } \\ \rho_{\mathrm{b}}\left(\mathrm{cm}^{-2}\right) & \text { Distribution of branching density } \\ b\left(\mathrm{~cm}^{-2} \mathrm{~d}^{-1}\right) & \text { Volumetric root branching rate } \\ b_{\mathrm{i} 1}\left(\mathrm{~d}^{-1}\right) & \text { Apical branching rate } \\ b_{\mathrm{i} 2}\left(\mathrm{~d}^{-1}\right) & \text { Branching angle } \\ b_{\mathrm{i} 3}\left(\mathrm{~cm}^{-2} \mathrm{~d}^{-1}\right) & \text { Adventitious branching rate } \\ e\left(\mathrm{~cm} \mathrm{~d}^{-1}\right) & \text { Root expansion rate } \\ g\left(\mathrm{~d}^{-1}\right) & \text { Root tropic rate } \\ 1,2 & \text { Subscript indicating root branching order }\end{array}$

\section{ACKNOWLEDGMENTS}

We thank Ian McNaughton, Jim Anderson and Jacqueline Thompson for their assistance and technical advice, and members of the Resource Capture Group at Scottish Crop Research Institute, Tracy Valentine and Peter Gregory, who 
provided valuable comments on the manuscript. The Scottish Crop Research Institute receives support from the Scottish Government Rural and Environment Research and Analysis Directorate (RERAD, Workpackage 1.7).

\section{REFERENCES}

Acock B. \& Pachepsky Y. (1996) Convective-diffusive model of two-dimensional root growth and proliferation. Plant and Soil 180, 231-240.

Aerts R., Boot R. \& van der Aart P. (1991) The relation between above- and belowground biomass allocation patterns and competitive ability. Oecologia 87, 551-559.

Amtmann A., Hammond J.P., Armengaud P. \& White P.J. (2006) Nutrient sensing and signalling in plants: potassium and phosphorus. Advances in Botanical Research 43, 209-257.

Avriel M. (2003) Nonlinear Programming: Analysis and Methods. Dover Publishing, New York.

Badri D.V. \& Vivanco J.M. (2009) Regulation and function of root exudates. Plant, Cell \& Environment 32, 666-681.

Bastian P., Chavarria-Krauser A., Engwer C., Jaeger W. \& Marnach S. (2008) Modelling in vitro growth of dense root networks. Journal of Theoretical Biology 254, 99-109.

Beemster G.T.S. \& Baskin T.I. (1998) Analysis of cell division and elongation underlying the developmental acceleration of root growth in Arabidopsis thaliana. Plant Physiology 116, 1515-1526.

Bonser A.M., Lynch J. \& Snapp S. (1996) Effect of phosphorus deficiency on growth angle of basal roots in Phaseolus vulgaris. New Phytologist 132, 281-288.

Chavarria-Krauser A. \& Schurr U. (2004) A cellular growth model for root tips. Journal of Theoretical Biology 230, 21-32.

Claassen N. \& Barber S.A. (1974) A method for characterizing the relation between nutrient concentration and flux into roots of intact plants. Plant Physiology 54, 564-568.

Clausnitzer V. \& Hopmans J. (1994) Simultaneous modeling of transient three-dimensional root growth and soil water flow. Plant and Soil 164, 299-314.

Crick J.C. \& Grime J.P. (1987) Morphological plasticity and mineral nutrient capture in two herbaceous species of contrasted ecology. New Phytologist 107, 403-414.

Doussan C., Pierret A., Garrigues E. \& Pagès L. (2006) Water uptake by plant roots: 2 - modelling of water transfer in the soil root-system with explicit account of flow within the root system - comparison with experiments. Plant and Soil 283, 99-117.

Drew D.A. (1975) Comparison of the effects of a localized supply of phosphate, nitrate, ammonium and potassium on the growth of the seminal root system, and the shoot, in barley. New Phytologist 75, 479-490.

Dupuy L., Fourcaud T. \& Stokes A. (2005a) A numerical investigation into the influence of soil type and root architecture on tree anchorage. Plant and Soil 278, 119-134.

Dupuy L., Fourcaud T., Stokes A. \& Danjon F. (2005b) A density based approach for the modelling of root architecture: application to Maritime pine (Pinus pinaster Ait.) root system. Journal of Theoretical Biology 236, 323-334.

Fitter A.H. \& Stickland T.R. (1991) Architectural analysis of plant root systems 2. Influence of nutrient supply on architecture in contrasting plant species. New Phytologist 118, 383-389.

Fitter A.H., Stickland T.R., Harvey M.L. \& Wilson G.W. (1991) Architectural analysis of plant root systems 1. Architectural correlates of exploitation efficiency. New Phytologist 118, 375-382.

Forde B.G. \& Walch-Liu P. (2009) Nitrate and glutamate as environmental cues for behavioural responses in plant roots. Plant, Cell and Environment 32, 682-693.

French A.P. (1971) Vibrations and Waves. Nelson, London.
Ge Z., Rubio G. \& Lynch J. (2000) The importance of root gravitropism for inter-root competition and phosphorus acquisition efficiency: results from a geometric simulation model. Plant and Soil 218, 159-171.

Gerwitz A. \& Page E.R. (1974) An empirical mathematical model to describe plant root systems. Journal of Applied Ecology 11, 773-781.

Gilroy S. \& Jones D.L. (2000) Through form to function: root hair development and nutrient uptake. Trends in Plant Science 5, 56-60.

Godin C., Costes E. \& Sinoquet H. (1999) A method for describing plant architecture which integrates topology and geometry. Annals of Botany 84, 343-357.

Grabarnik P., Pagès L. \& Bengough A.G. (1998) Geometrical properties of simulated maize root systems: consequences for length density and intersection density. Plant and Soil 200, 157167.

Hackett C. (1971) Relations between the dimensions of the barley root system: effects of mutilating the root axes. Australian Journal of Biological Science 24, 1057-1064.

Hackett C. \& Rose D. (1972) A model of the extension and branching of a seminal root of barley, and its use in studying relations between root dimensions. I. The model. Australian Journal of Biological Science 25, 669-679.

Heinen M., Mollier A. \& De Willigen P. (2003) Growth of a root system described as diffusion. II. Numerical model and application. Plant and Soil 252, 251-265.

Hodge A. (2004) The plastic plant: root responses to heterogeneous supplies of nutrients. New Phytologist 162, 9-24.

Hodge A. (2009) Root decisions. Plant, Cell \& Environment 32, 628-640.

Hodge A., Robinson D., Griffiths B.S. \& Fitter A.H. (1999) Why plants bother: root proliferation results in increased nitrogen capture from an organic patch when two grasses compete. Plant, Cell \& Environment 22, 811-820.

Jourdan C., Rey H. \& Guédon Y. (1995) Architectural analysis and modelling of the branching process of the young oil-palm root system. Plant and Soil 177, 63-72.

Leveque R.J. (2002) Finite Volume Methods for Hyperbolic Problems. Cambridge University Press, Cambridge, UK.

Lynch J. (2007) Roots of the second green revolution. Australian Journal of Botany 55, 493-512.

Meister A. \& Struckmeier J. (2002) Hyperbolic Partial Differential Equations: Theory Numerics and Applications. Friedrich Vieweg \& Sohn Verlag, Braunschweig.

Morgan J.A.W., Bending G.D. \& White P.J. (2005) Biological costs and benefits to plant-microbe interactions in the rhizosphere. Journal of Experimental Botany 56, 1729-1739.

Nichol S.A. \& Silk W.K. (2001) Empirical evidence of a convectiondiffusion model for $\mathrm{pH}$ patterns in the rhizospheres of root tips. Plant, Cell \& Environment 24, 967-974.

Pages L. (1995) Growth patterns of the lateral roots of young oak (Quercus robur) tree seedlings. Relationship with apical diameter. New Phytologist 130, 503-509.

de Reffye P. \& Houllier F. (1997) Modelling plant growth and architecture: some recent advances and application to agronomy and forestry. Current Science 73, 984-992.

Remans T., Nacry P., Pervent M., Filleur S., Diatloff E., Mounier E., Tillard P., Forde B.G. \& Gojon A. (2006) The Arabidopsis NRT1.1 transporter participates in the signaling pathway triggering root colonization of nitrate-rich patches. Proceedings of the National Academy of Sciences 103, 19206-19211.

Robinson D. (1996) Resource capture by localized root proliferation: why do plants bother? Annals of Botany 77, 179-186.

Rose D. (1983) The description of the growth of root systems. Plant and Soil 75, 405-415. 
Silk W.K. (2002) The kinematics of primary growth. In Plant Roots, the Hidden Half (eds Y. Waisel, A. Eshel \& U. Kafkafi), pp. 113-126. Marcel Dekker, New York.

Svistoonoff S., Creff A., Reymond M., Sigoillot-Claude C., Ricaud L., Blanchet A., Nussaume L. \& Desnos T. (2007) Root tip contact with low-phosphate media reprograms plant root architecture. Nature Genetics 39, 792-796.

Walk T., Jaramillo R. \& Lynch J. (2006) Architectural tradeoffs between adventitious and basal roots for phosphorus acquisition. Plant and Soil 279, 347-366.

White P.J. (2001) The pathways of calcium movement to the xylem. Journal of Experimental Botany 52, 891-899.

White P.J., Whiting S.N., Baker A. \& Broadley M.R. (2002) Does zinc move apoplastically to the xylem in roots of Thlaspi caerulescens? New Phytologist 153, 201-207.

White P.J., Broadley M.R., Greenwood D.J. \& Hammond J.P. (2005) Genetic modifications to improve phosphorus acquisition by roots. Proceedings of The International Fertiliser Society 568, IFS, York, UK.

Wilkinson S. \& Davies W.J. (2002) ABA-based chemical signalling: the co-ordination of responses to stress in plants. Plant Cell and Environment 25, 195-210.

Yang C.-H. \& Crowley D.E. (2000) Rhizosphere microbial community structure in relation to root location and plant iron nutritional status. Applied and Environmental Microbiology 66, 345-351.

Zhang H. \& Forde B.G. (1998) An Arabidopsis MADS box gene that controls nutrient-induced changes in root architecture. Science 279, 407-409.

Received 25 August 2009; received in revised form n/a; accepted for publication 22 October 2009

\section{SUPPORTING INFORMATION}

Additional Supporting Information may be found in the online version of this article:

Text S1. Conservation of meristem number.

Text S2. Analytical solution of one-dimensional meristem dynamics using the method of characteristics.

Please note: Wiley-Blackwell are not responsible for the content or functionality of any supporting materials supplied by the authors. Any queries (other than missing material) should be directed to the corresponding author for the article. 